\title{
Is it Time to Look at What We Do?
}

\author{
By Scot Morris, OD
}

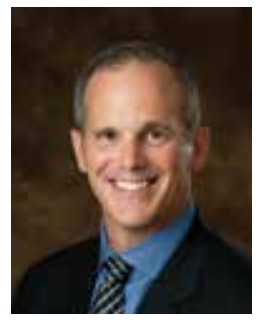

Dr. Scot Morris owns an independent practice in Denver, Colorado. He is also the Co-founder of $4 E C P s-$ a business resource company for Optometrists. 4ECPs has three divisions, Jobs - Training - Marketing. Check out more information at www.4ECPs.com. Scot is also editor of Optometric Management, speaker, author, consultant and business innovator. Correspondence may be directed to scot@mecace.com.

$\mathrm{W}$ e often go about our day and do the same thing over and over again. Let's call it routine. But is it the best and most efficient way of doing things or just "what we do?" Let's look at this from a consumer perspective. How long should an exam take? Fifteen minutes, 30 minutes, 45 ? For all of us, time is the one thing we can't get enough of or get back and it is our most valuable resource. Are we valuing that of our consumers, and our own?

I want to challenge each of you reading this to work an hour less a day. That's right, one hour less. What would you do with that hour? Do I have your attention now?

Let's learn how to "find" that hour. If you are seeing 15 patients a day and you can "save" 4 minutes per patient, then you now have an extra hour. That is only four minutes. In most instances, practices could improve their efficiency by $20-40 \%$ (8-12 minutes) and improve their bottom lines substantially by just analyzing what each and every person in the office actually does every day. This process is called workflow efficiency analysis.

All businesses have a workflow process. Many businesses have no idea what their process is though. There are approximately 102 steps to get a patient from the time they schedule an appointment until they are seen again the following year, and this doesn't include any marketing, human resources, inventory management, or operational tasks. Each of these steps is an opportunity lost or gained to educate your customer, to sell something, and an opportunity to make an impression-good or bad. If you really want to provide better care, more efficient service, and improve your bottom line, this exercise will be one of the most eye opening and beneficial experiences that you will ever undertake as a business person. By thinking like a business person, not a doctor, you will start to think about how each step of the process works.

So how do we improve both our efficiency and our effectiveness?
This takes four steps.

STEP 1: Identify where you are now. How long does it take you to currently see a patient? We call this DDD (Door to Dr. Done). First of all, write down what you think it is. Let's call this the estimate. Now figure out what the "real" number actually is. Document what time a patient arrives and what time the doctor is done and the exam moves to the retail part of the visit. Next, determine what your real ratios are. The best way to evaluate this is to measure your Encounters per Hour. This is determined by taking the Total \# of Patients/ Hours worked in a given time period.

STEP 2: Identify what you do now. The next step is to identify each of the approximately 100 steps in your consumer's experience. Identify the processes that occur at each step of the consumer process in your office. Start slow. Ask each person in your office to list specifically each step that your front desk performs. Next, have each person in the office list in order which process occurs first, second, and so on. Have them do this individually. Do it yourself. Then collect and combine all the answers. Prepare to be amazed. Don't be surprised if everyone in the office gives completely different answers. The graph below gives an example of the various steps that may happen just during the "appointment and check-in" process. Look at it as a challenge to get everyone on the same page. This process will take a few weeks. This will take 6-8 weeks to complete.

STEP 3: Process Streamlining. Now break down each step and what is actually being done and said. If the process doesn't have a purpose that educates, sells, or improves efficiency, get rid of that step. Now repeat this step for each department (reception pre-test, provider, optical, contact lens, check-out, billing). Determine who says what. Who does what. What do they do it or say? Who do they say it to? 
STEP 4: Communication Review \& Process Refinement (scripts and protocols) Have your staff write scripts of what they actually say to the consumer during each step. If they are not educating or selling, then you need to change what is being said. Then decide if each step could be done more efficiently or eliminated altogether. This process may take a month or so. Once you are done with this step move to pre-test, the doctor's exam, the optical, the contact lens area, check-out, and billing. This process does take a while but the sea changes in efficiency and profitability are well worth it. In essence, at each point of the process, we need to decide how the following things are being presented. Consider each of the points in the table to the right when assessing the scripts for each step.

\section{Communication Considerations}

1. What are we trying to accomplish? (Our goal)

2. What are we trying to sell or promote?

3. What is their need or want? How do we find this out?

4. How can we orient them to what we want to talk about?

5. What are the potential barriers to communication

6. Is that what we are really saying?

7. Do they understand it? How do you know?

8. How can we follow-up?

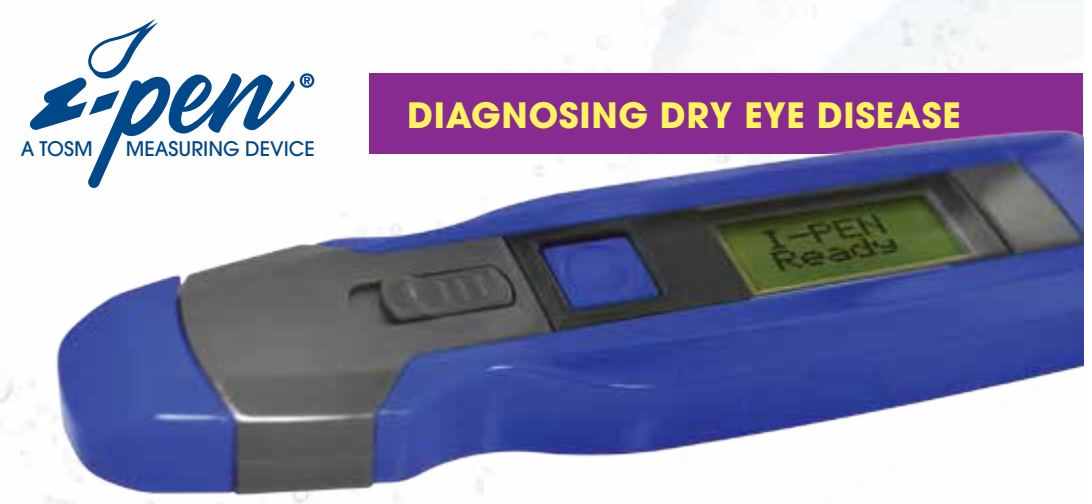

Announcing a Completely New Approach to

Diagnosing Dry Eye Disease by Measuring Tear Osmolarity 